\section{Citalopram-resistant depression: cognitive therapy and medication similarly effective as second line treatments}

\section{QUESTION}

Question: Cognitive therapy or pharmacotherapy as secondline for people with citalopram-resistant depression - which is more effective?

Patients: 304 out-patients with non-psychotic major depressive disorder (clinically established diagnosis verified through DSM-IV criteria) enrolled in the Sequenced Treatment Alternatives to Relieve Depression (STAR*D) study who did not achieve remission (remission defined as Quick Inventory of Depressive Symptomatology score $\leqslant 5$ ) with first-line citalopram treatment. This analysis included only those people who agreed to be randomised to treatment strategies (augmentations, switches or both) which would allow cognitive therapy to be compared with pharmacotherapy.

Setting: Primary care and psychiatric care practice settings across the USA; recruitment July 2001 to April 2004.

Intervention: Participants who did not achieve remission were randomised to one of seven second-line treatments: ongoing citalopram with sustained-release bupropion, buspirone, or cognitive therapy (augmentation strategies; $n=182$ ); or discontinuing citalopram and starting therapy with sertraline, sustained-release bupropion, extended-release venlafaxine, or cognitive therapy (switch strategies; $\mathrm{n}=122$ ). In this pragmatic RCT, data were analysed in strata according to what treatment options participants considered acceptable. Only those strata which would allow a direct comparison of cognitive therapy (as augmentation or switch) versus pharmacotherapy were included. These were strata where participants had said they would accept "only augmentations", "only switches", "any treatment (except switch to cognitive therapy)", "any treatment", "any treatment (except medication augmentation)", "any treatment (except switch to medication)".

Outcomes: Depression remission (score $\leqslant 7$ on the Hamilton Rating Scale for Depression (HAM-D)) assessed at the beginning and end of treatment through telephone interviews; time to remission.
Patient follow-up: Unclear; only 27/101 (27\%) of people randomised to cognitive therapy (augmentation or switch) completed the full 16 sessions of scheduled treatment.

\section{METHODS}

Design: Randomised controlled trial.

Allocation: Unclear.

Blinding: Single blind (assessors blinded).

Follow-up period: 12 weeks.

\section{MAIN RESULTS}

There was no difference between augmentation with cognitive therapy and augmentation with medication in rate of remission from depression (remission rate: 23\% with cognitive therapy vs $33 \%$ with medication; $p=0.1967$ ). Augmentation with medication reduced time to remission $(p=0.022)$. There was no difference between switch to medication and switch to cognitive therapy in remission (remission rate: $25 \%$ with cognitive therapy vs $28 \%$ with medication; $p=0.6881$ ). There was also no significant difference between groups in the time to remission $(p=0.9350)$. The number of people experiencing side effects was greater with medication switch, but discontinuation because of intolerance to treatment was not significantly different between switch arms $(17 \%$ with cognitive therapy vs $27 \%$ with medication; $p=0.2330$ ).

\section{CONCLUSIONS}

There were no appreciable differences between cognitive therapy and pharmacotherapy as second-line treatment in citalopram-resistant depression, but the study may have been underpowered to detect differences.

\section{ABSTRACTED FROM}

Thase ME, Friedman ES, Biggs MM, et al. Cognitive therapy versus medication in augmentation and switch strategies as second-step treatments: a STAR*D report. Am J Psychiatry 2007; 164:739-52.

Correspondence to: Dr Thase, University of Pittsburgh Medical Center, 3811 O'Hara Street, Pittsburgh, PA 15213-2593, USA; thaseme@upmc.edu

Source of funding: National Institute of Mental Health.

- Additional notes are published online only at http://ebmh.bmj.com/content/vol11/ issue2
$T$ his study, one of many from STAR*D, seeks to answer questions about real world treatments and contexts. The findings - that CBT is equivalent to pharmacotherapies as a second step after non-remission with citalopram - is plausible and reassuring. A number of factors erode the study's potential for validity from a pure efficacy perspective: very few agreed to randomisation to CBT and only $25 \%$ completed the allotted 16 sessions. However, these limitations actually support the generalisability and effectiveness of the findings. For instance, the study provided medications free, but co-payments were required for CBT, and it is possible that some subjects may have had insurance plans that would have required full payment for therapy, dissuading some from opting for potential randomisation to CBT.
Patients could be switched to CBT monotherapy or could have CBT added to pharmacotherapy; those in the switch pathway were less likely to agree to CBT, as that also involved terminating treatment with the original physician who began their depression treatment. Furthermore, individuals assigned to CBT received therapy from adequately trained and monitored, but non-expert, CBT therapists who usually worked at a treatment setting different from the one where the subject saw the treating physician. Thus, what was tested was the effectiveness of what is a real world scenario: what happens when you send the patient away to see an average CBT therapist at another site? The answer is that many patients do not want to go, many do not complete treatment, but overall they do as well as if they had stayed in your office and received another pharmacotherapy. Both for the clinician and the patient, it is very helpful to know that there is true clinical equipoise about the next treatmentmedication or CBT-after failure of the first antidepressant. Furthermore, the answer is that the next treatment has about a 30\% chance of hitting remission, with medication reaching that point slightly sooner.

\section{Sagar V Parikh, MD, FRCPC}

University Health Network and University of Toronto, Toronto, Canada

Competing interests: None. 